\title{
An anthropomorphic robotic end effector
}

\author{
Richard M. Crowder \\ Depts. of Electrical and Mechanical Engineering, University of Southampton, Southampton SO9 5NH, UK
}

\begin{abstract}
Crowder, R.M., An anthropomorphic robotic end effector, Robotics and Autonomous Systems, 7 (1991) $253-268$.

Research into the development of artificial mechanical hands for prosthetic applications has been conducted over many hundreds of years. Dexterous mechanical hands are now being applied to advanced robotic applications, including tele-operated manipulators. The successful application of dexterous mechanical hands depends on the development of suitable mechanisms, actuators and control strategies. Recently the requirement arose for a manipulator to fit and operate within a standard glove box glove. The design and development of a fully anthropomorphic dexterous end effector for the manipulator is discussed in this paper. To arrive at the final design a study of the operation of the human hand was undertaken. The end effector consists of four mechanically adaptive fingers and a thumb. The mechanism developed for the fingers will simulate the curl of the human finger. The complete finger mechanism requires only a single actuator to produce fully the required motion, leading to a compact design for the end effector. To gain the full operational advantage of the dexterous hand a control strategy has been developed based on global sensing and a hierarchical control structure. The results of handling trials are presented.
\end{abstract}

Keywords: Manipulators; Mechanisms; Dexterous hands; Sensors.

\section{Introduction}

The commercial industrial robot typically consists of an end effector attached to the end of a multi-degree of freedom mechanical arm. The purpose of the arm is to position and orientate the end effector and then act as a support while the required task is performed. The end effector can be either a tool, for example a welding head, or a gripper to hold other tools or the work piece. The most widely used form of robot gripper consists of two or more movable rigid fingers working in

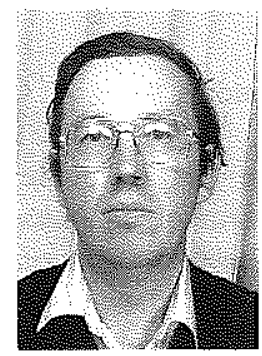

Richard M. Crowder was awarded his $\mathrm{BSc}$ and $\mathrm{PhD}$ from the University of Leicester. Following employment at Maudsley Ltd. and L.P.E. (Electronics) Ltd. he moved to the University of Southampton in 1984 to take up a lectureship in Robotic Actuators and Manipulators. This is a joint appointment between the Departments of Electrical Engineering and Mechanical Engineering. His present research includes robotic end effectors, multiarmed robot simulation and aircraft actuators. opposition, this however limits the sizes and shapes of objects that can readily be grasped. The orientation of the gripper relative to the object is crucial if the grip is to succeed, and can only be modified by the manipulator. This restriction leads to the requirement for a gripper that can grip a wide range of different shaped objects from various orientations. If the end effector has the ability to adapt to the shape of the gripped object and manipulate it within the confines of the gripper, the resultant end effector is commonly termed a dexterous hand. The earliest use of mechanical hands was for prosthetic applications of which examples have been recorded as far back as fourteenth century [1]. The current level of technology is such that the prosthetic hand is limited to gripping objects, the detailed manipulation is then the function of the remaining arm joints of the amputee. Efficient hand operation currently depends on direct visual information to guide the hand and not from the dexterity of the prosthesis itself. For this reason the prosthetic hook is still widely used relative to the more cosmetic, but less 
flexible, anthropomorphic hands available. Recent work [2], has shown that the addition of touch and slip sensors to a commercial prosthetic hand gives a considerable improvement in dexterity which is achieved without a corresponding increase in conscious effort from the wearer.

The requirement of the nuclear, space and subsea industries has caused the development of a wide range of tele-operated and master-slave manipulators, because of the hazards to a human operator working in these environments [3]. The addition of a dexterous mechanical end effector to these manipulator systems would greatly enhance their performance. Over the years a number of non-prosthetic dexterous hands have therefore been developed which are based on the use of multi-jointed articulated designs that mimic the motion of the human finger. Details of a number of significant designs have been published, including the Utah/MIT hand [4], which has four fully articulated fingers. Conversely, the hand developed by the University of Standford and JPL [5], is based on a three-finger design. These and other hands have been developed primarily as tool for research into artificial intellegence and gripping strategy. Within the remote handling field the use of the dexterous end effector is still limited due to the difficulty in developing suitably rugged mechanical and sensing systems required by these applications. If the use of the dexterous end effector was more widely applied to more traditional industrial applications, its inherent ability to adapt to the object being gripped would reduce the inventory of specialist grippers and hence lead to an increase in the application of robotic systems.

\section{Project background}

Within industry, world wide, the awareness to the hazards of radioactive, chemical and biological materials has increased rapidly over recent years. This has led to a reduction in the amount of exposure to which an operator can be subjected. The result is an urgent requirement to remove numan operators from the hazardous environments and hence, this leads to an increasing reliance on robotics and dedicated automated sysems. However, due to the nature of some processes here is still a requirement for a high degree of nanual intervention, due to the complexity of the task and/or its unsuitability for direct automation.

A large number of these processes are carried out within a standard industrial glove box, the operator is protected by the use of heavy duty synthetic rubber gloves. One solution that would reduce the hazard to the operator is for a manipulator to have access to the hazardous environment using the operators' glove. This however requires that the manipulator and its end effector are capable of operating within the glove and in particular the end effector has to be fully anthropomorphic. In spite of the complexity of the manipulator this approach has a number of significant advantages over an individual robot per glove box. Firstly the manipulator is ouside the hazardous environment and hence can easily be moved between glove boxes without distroying the integrity of the glove box environment, and secondly the number of manipulator systems required to equip a facility is minimised.

Recently, this particular application arose within the UK nuclear industry. Initial approaches were made to the manufacturers of prosthetic limbs, but it was realised at an early stage that the level of technology within this industry was not at that time capable of meeting the design requirements for such a glove box manipulator. As a result of this requirement the Department of Electrical Engineering at the University of Southampton has developed the whole arm manipulator, a six- axis glove box manipulator. The significant feature of the manipulator is the multifingered hand incorporating mechanically adaptive finger mechanisms.

This paper discusses the design and development of an anthropomorphic mechanical hand that is capable of fitting and operating within a human sized glove manufactured from synthetic rubber. A previously published paper has given an overview of the complete system [6].

\section{The design of the mechanical hand}

In the preparation of the outline design for the anthropomorphic end effector, a number of areas had to be considered, including the operation of the human hand both in and outside the glove. Only then could a specification for the mechanical design of the anthropomorphic end effector for 
the whole arm manipulator be developed. In parallel with the mechanical design, the control strategies for the hand had to be identified. Due to the complexity of the tasks that have to be performed, an easy-to-use operator interface is required for the whole system therefore reducing the work load on the operator.

\subsection{The glove}

The objective of this project was the design of a manipulator capable of performing a wide range of tasks inside a glove box whilst being enclosed within a standard glove The integrity of the glove box must not be damaged during normal operation or in the changeover between the manipulator and a human operator. This ensures that neither the manipulator nor the local environment become contaminated with the contents of the glove box.

Details of the hand of the glove into which the whole arm manipulator is designed to operate is

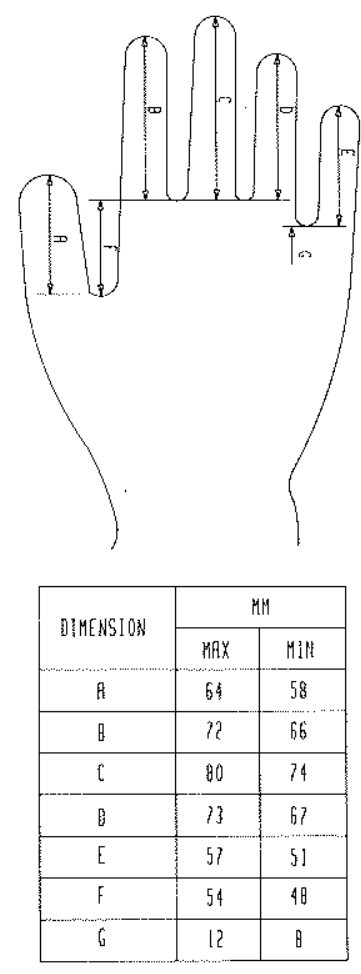

Fig. 1. Dimensions of the hand of the synthetic rubber glove. The variations are due to different moulds used during manufacture. shown in Fig. 1, the overall length of the glove is $780 \mathrm{~mm}$. Due to the position of the glove when installed in the glove box the maximum internal reach of the manipulator is limited to $500 \mathrm{~mm}$ from the glove box face, this is equilvalent to the operator inserting his arm into the glove up to the elbow. Based on this criterion the length of the forearm of the manipulator and its relationship to the elbow and hand is fixed. The size of the hand is similarly determined by the size and location of the glove's fingers together with the position of the forearm. The length of the individual fingers are determined from the datum position located at the crotch of the third and fourth fingers. The minimum inter-finger spacing was determined by the thickness of the glove within the crotch. To allow for satisfactory operation the finger spacing was set to $4 \mathrm{~mm}$. The major dimensions of the hand and the manipulator are shown in Fig. 2. To prevent contamination of the operator in case of a glove puncture the inside of the glove box is held at negative pressure of $500 \mathrm{Nm}^{-2}$ (5 Mbar), at this pressure differential the glove becomes quite rigid.

\subsection{Review of human hand operation}

The structure and operation of the human hand is one of the significant differences between humans and the other members of the ape family. The human hand consists of the wrist (the carple bones), the palm (the metacarple bones) and the fingers (phalanges). The palm and fingers of the human hand together have twenty degrees of freedom; two in the knuckles, one in each of the other finger joints, three in the thumb and one in the palm. However, the most characteristic feature of the human hand is its truely opposable thumb whereby the thumb can rotate and oppose any of the fingers to pinch an object. It is this feature that gives the human its superb dexterity with tools. The operation of the hand may be considered either as static (gripping) or as a dynamic operation (manipulation). Schlesingert [7], indentified six hand configurations that characterise the static gripping functions of the human hand, namely:

(1) Cylindrical grasp - the grip used to enclose a cylindrical object.

(2) Spherical grasp - used when grasping a spherical object. 
that the correct shape will be achieved when the mechanical finger is curled. The finger joint is one of the few in the human body that requires a maximum movement of $90^{\circ}$ between components.

(4) In a human hand the thumb is opposable so that pinch grip can occur between the thumb and any of the four fingers either to the sides or to the tips. If this was to be achieved in the mechanical hand, the thumb abduction would require control. This motion has since been achieved at the University of Southampton on a prosthetic hand, but due to the size constraint of the anthropomorphic mechanical hand required in this application, this movement could not be incorporated. In the final design the tip of the index finger and tip of the pseudo-opposable thumb are used to provide the pinch grip.

The effect of these considerations is the reduction of the number of independent degrees of freedom that require control. To pinch an object the thumb and index finger require independent control. For the hand's power grip, this being a cylindrical grasp, all four fingers are used. It is therefore possible to fully control all the required functions of this robotic anthropomorphic hand with only three actuators, thumb, index finger and the remaining fingers as a coordinated group.

The specifications of whole arm manipulator require that the maximum load capability of the system was to be $5 \mathrm{~kg}$ load in the hand, irrespective of hand orientation and object shape. Additionally, the applied finger pressure was to be equal to that of a human, typically $30 \mathrm{~N}$ at the tip.

\section{The Southampton mechanical hand}

The mechanical hand can be considered to consist of three separate areas: the fingers and their associated mechanisms, the thumb and the palm. The satisfactory operation of the hand depends upon the ability of the fingers to curl around an object of unknown shape, therefore the fingers and their associated mechanisms will be considered first.

\subsection{The finger mechanism}

The basic design requirement of the fingers and hence their mechanism is that each finger is to consist of three segments, the maximum relative movements between segments to be $90^{\circ}$, and the upper two finger sections are required to produce a coordinated curling motion. The finger must have a rigid structure for the load requirements and be capable of accepting touch sensors and any associated wiring. The construction of the fingers is based principally on the use of side plates and cross pivots. This mode of construction gives the maximum clear space within the profile of fingers for the mechanical linkages and sensors.

The correct selection of the finger actuators is of crucial importance to the operation of the whole arm manipulator. The main requirement of the manipulator is that it is able to operate within the glove. A design study clearly showed that the maximum clearance between the glove and the arm of the manipulator was $10 \mathrm{~mm}$. When the manipulator rotates within the glove, the glove will become twisted which reduces the clearance. This movement had in no way to interfere with the operation of fingers, even at minimum clearance.

A number of dexterous hand systems are based on tendon actuators [4,5], where each finger joint is connected to a remote actuator by a flexible cord or tendon. To achieve full joint motion a minimum of two tendons are required per joint, in the case of the whole arm manipulator, a minimum of twenty-six individual tendons, actuators and control loops would be required for the proposed hand. The advantage of this approach is that the actuators are remote from the hand and hence reduce the overall inertia by removing mass from the end of the manipulator. The clearance restriction imposed by the movement of the manipulator within the glove was such that the use of external actuators together with tendons was not a practical proposition for this application. Furthermore, the use of hand mounted pneumatic and hydraulic actuators was eliminated for identical reasons. It was concluded that for this hand to operate satisfactorily, electric actuators would have to be located within the palm area of the hand. As the physical size of the palm limits the number of actuators to a maximum of three, this design solution is only possible if the motion of the fingers could be controlled by their mechanical linkage. An advantage of this design is the high reliability of electric motors, this was an important consideration as the manipulator is intended for continuous industrial operation. 


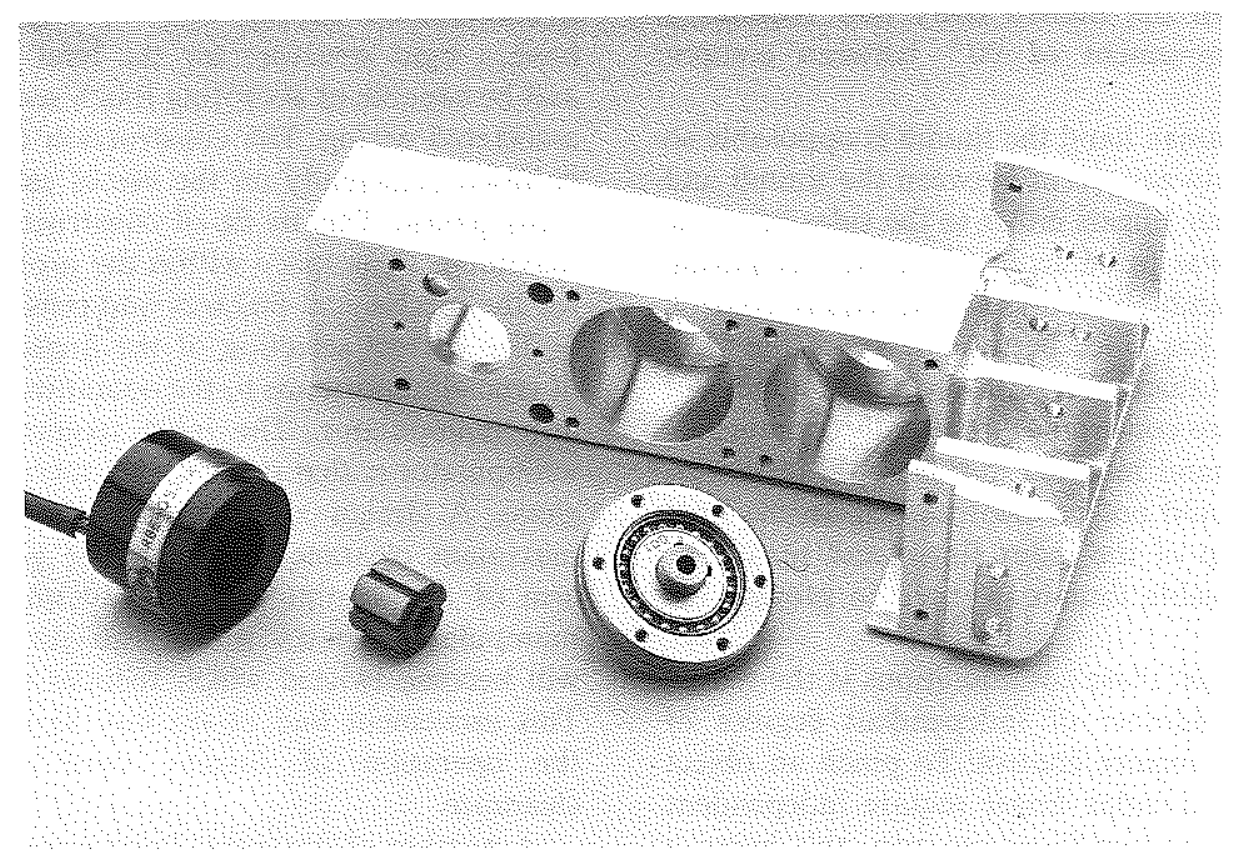

Fig. 3. The central core of the palm, with the motor and gearbox. (The motor armature is to the left.)

The requirements for the actuator are high power, small size and high reliability. The combination selected was a brushless dc motor (Inland type RBE 00500) driving a high-ratio harmonic gear box (Harmonic Drive type HDUF 8-100-2). The selected motor and gearbox combination is capable of producing a continuous output torque of $1.9 \mathrm{Nm}$ at $70 \mathrm{rpm}$. In the hand the frameless dc brushless motors are mounted within the palm block, with the harmonic drives and the rotary-tolinear mechanisms fitted to each side of the palm, supported by the outer face plates. The motor and gearbox together with the central core of the palm is shown in Fig. 3.

\subsubsection{Principle of finger operation}

The University of Southampton has developed a range of adaptive finger mechanisms which only require a single drive input $[9,10]$. The mechanism design for the hand of the whole arm manipulator was based upon this work. In terms of the actual finger operation, a mechanical finger can be designed to operate by one of two basic methods, either the mechanism can be designed to give a con-

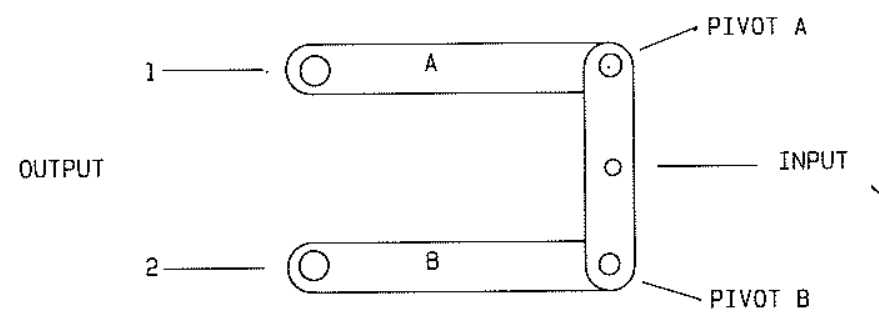

Fig. 4. The basic equalising bar. 


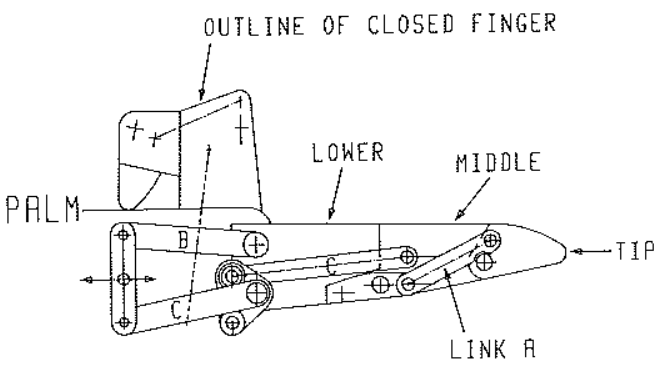

Fig. 5. The operating mechanism for the index finger.

trolled relative movement between each finger segment and the knuckle pivot or a differential mechanism giving independent movement between each finger section and the knuckle. The selected mechanism has to allow the hand to grasp objects of various shapes and sizes. The first mechanism will give a fixed trajectory of finger motion irrespective of the gripped object, hence for this application the differential mechanism was selected as it allows the finger to have the required wrap-around capability.

The fundamental operating principle of the finger mechanism is based on the use of an equalising bar (Fig. 4). When the pivot at the centre of the equaliser bar moves along the path shown, with links $A$ and $B$ free to move, the applied force will be transmitted to the outputs 1 and 2. The magnitude of the output force is half that of the applied force. If however output 1 becomes fixed for any reason, the equaliser bar will rotate around pivot $A$ and all the input force will be transmitted via link B. This operating principle can be clearly seen in Fig. 5, the index finger.

The index finger consists of three segments (lower, middle and tip) pivoted together. The upper segment is linked to the lower segment by link $A$, so that any motion of the middle segment will cause the upper segment to move. This linkage will produce the required curl motion. The lower and middle segments are individually connected by links ( $B$ and $C$ respectively) to either end of the equaliser bar. The link to the upper segment is split by a crank, this geometry produces a poorer mechanical advantage compared with the lower segment and link B. Finally, the equaliser bar is connected by a slider and crank mechanism to the rotary actuator.

In the rest position the finger is considered to be in the fully extended position. To close the finger the equaliser bar is driven to the left. Due to the difference in the mechanical advantages between links $\mathrm{B}$ and $\mathrm{C}$ and their respective segments the whole finger will preferentially rotate around the pivot of the lower segment and the palm. The design of the mechanism is such that the finger remains straight while it rotates around this pivot, which is located behind the crank in Fig. 5. The rotation of the complete finger will continue until such time as the lower segment is stopped either by its mechanical limit or by an external object. As the lower segment and link B cannot now move the closing force is transferred to the middle segment, via link $\mathrm{C}$, thus causing the upper two segments of the finger to curl over and complete the grip around the object. The outline of the closed finger is also shown in Fig. 5. This design ensures that the tip just clears the palm on closure. The resultant finger motion is similar to that of a human finger and is described as being "tip driven", as the finger tip effectively leads the motion. On motor reversal the finger will open and return to the rest position. Due to the floating nature of the upper two segments a spring return is required, in the case of the whole arm manipulator this is provided by the elasticity of the synthetic rubber glove.

The other three fingers are operated simultaneously, but independently of the index finger, by the mechanism shown in Fig. 6. The upper link, B, from the equiliser bar is directly coupled to the lower segment of the fourth finger. The lower segments of the other fingers are directly coupled to the fourth finger, hence the motion of all the lower segments will stop when any of the three segments touches an end stop or an object. This will cause the equiliser bar to transfer the closing force to the upper segments which will then curl

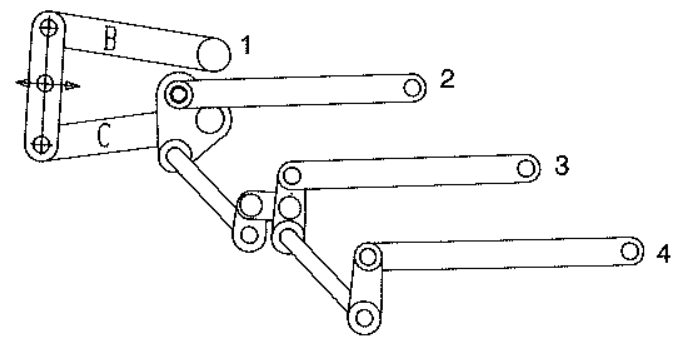

Fig. 6. Operating mechanism for second, third and fourth finger. Point 1 is connected to the lower link of the fourth finger. Points 2, 3 and 4 are connected to the middle links of the fourth, third and second finger respectively. 
round the object, until at least one is in contact with the object. The design of the hand ensures that there are at least three fingers in contact with an object at any time.

The action of the finger mechanism primarily depends on the differences between the mechanical advantages of the output links of the equaliser bars of the mechanisms shown in Figs. 4 and 5 . The differences in the frictional forces between the links and the stiffness of the glove will also effect the motion of the fingers. It was therefore necessary to trim the mechanism to achieve optimum performance; this was achieved by moving the central pivot point of the equaliser bar away from the geometric centre of the bar, in the case of the index finger a $5 \mathrm{~mm}$ offset was found to be required.

\subsection{The thumb}

As discussed previously the thumb is designed to make contact with the tip of the index finger to form a pinch. In this anthropomorphic mechanical hand the thumb is jointless and is directly coupled to the output of its motor/gearbox assembly. The shape of the thumb and its actual location relative to the index finger are designed to allow a tip-to-tip pinch to be made when the plane of the pinch is normal to the palm's surface.

\subsection{The construction of the hand}

All the finger mechanisms together with the actuators are contained within the profile of the hand. To achieve a rigid structure the hand is built around a central ' $T$ ' shaped block, this is shown in Fig. 3. The motors are located within this block, the mass of material is used to improve the thermal characteristics of the motors. The field windings of the motors are glued in place when the unit is assembled. The finger mechanisms are positioned on either side of the ' $\mathrm{T}$ ' block. The cross piece of the ' $T$ ' contains the individual pivot points for the fingers, as in the human hand those for the fourth finger are located behind the others.

Considerable care was taken in the selection of materials used to construct the hand and special attention was paid to the strength and wear characteristic of the materials. Aircraft grade aluminium was used for structural elements, with

Fig. 7. The constructed hand, attached to the arm. The gearbox and shider mechanism for the second, third and fourth finger can be seen behind the lower side plate. 
bronze for the finger mechanisms. In certain locations stainless steel was used where high wear was predicted. During the design stages and building of the hand considerable care was taken to minimise the chances of glove puncture, largely through elimination of all possible sharp corners and points where the glove could be trapped.

The main features of the hand can be clearly seen in Fig. 7.

\section{Hand control strategy}

The mechanical design is such that only three motors need to be controlled to fully operate the hand. But as a unique motor position does not correspond to a unique finger position due to the mechanical design of the finger mechanisms, the development of a control strategy was of crucial importance to the project. The control strategy of the hand is based on that originally developed for the prosthetic hand work [2], namely that of a hierarchical control structure coupled with a small number of high-level input commands. The basic principle of this form of controller can be seen in Fig. 8. Within the system there are three basic levels:

Level 1. This contains the basic motor speed and torque control loops. The inputs to this level are the speed and torque requirements of the selected motors. Provision is made for touch sensing in this level, this may be derived from the motor current and the positional error as well as sensing elements mounted on the finger tips.

Level 2. The main hand control algorithms that take the operator's commands and determine the required hand motions are found at this level. The posture controller will determine which fingers

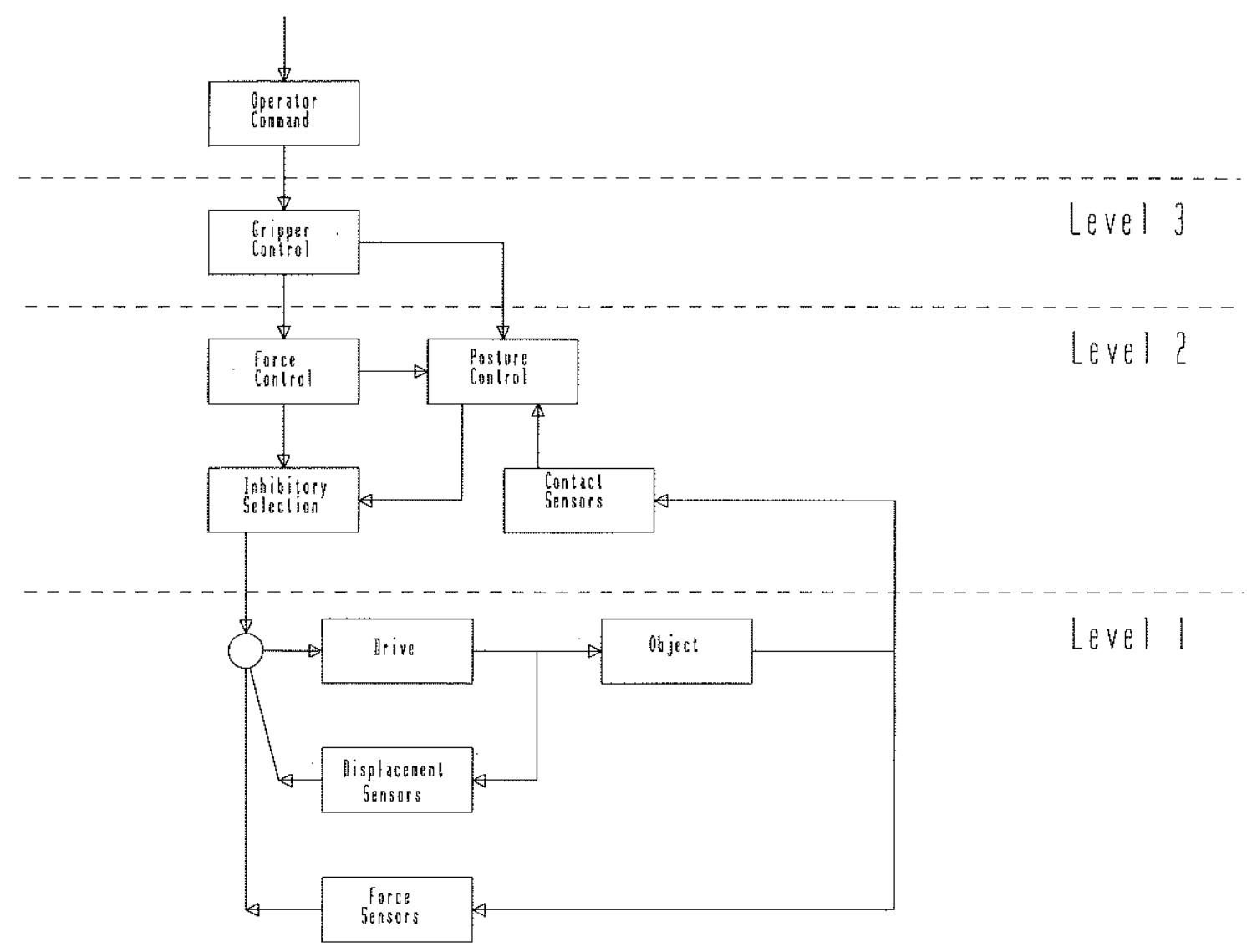

Fig. 8. The hierarchical control structure as used in the hand for the whole arm manipulator. 
have to be activated. The force controller calculates the required motor speeds and torques. Logic at this level will determine wheater a movement is valid.

Level 3. The upper level takes the required operator commands and converts them into the commands required by the second level.

The gripping of an object by a dexterous hand is an area of considerable importance, but however is not fully understood. The problem is complicated by the fact that the object has to be moved once grasped, but due to the unstructured environment within which the hand has to operate, there is little or no control over the object's location and orientation relative to the hand. In a successful grip the applied mechanical constraints will restrict the movement of the object. In the control of the hand for the whole arm manipulator two areas have to be addressed, firstly controlling the hand to achieve a grip and secondly its monitoring to ensure that the grip does not fail. To achieve satisfactory operation the hand must operate within a force feedback loop, the hand must therefore have sensors, either global or localised. Global sensing is the measurement of the total force exerted on the finger, while local sensing is the measurement of the forces at one particular point, namely touch sensing [11].

The philosophy on which the control of the hand is based is by the use of three basic operating configurations touch, pinch and grip, together with a home position. These were determined as a result of the work on the prosthetic hands. As the whole arm manipulator is a tele-operated system, the operator will have direct control over the configuration selected to perform the required tasks. The home position is defined as the position where all the fingers are in the outstretched position with the thumb parallel to the palm. This is the configuration required to load the arm into the glove. Selection of the touch mode will cause only the first finger to move, pinch will move the thumb and index finger in opposition. The selection of the grip mode will cause the thumb and all four fingers to form a cylindrical power grip. These modes can be cancelled as required by asking the hand to relax, causing the hand to open in a controlled manner.

\subsection{The objectives of sensing}

The stiffness of the mechanism determines what force will be applied to an external object when the link is deflected from its nominal position. The artificial stiffness of the finger is controlled by the applied motor torque, hence for satisfactory control a measure of the stiffness is required. A direct measure of the applied torque can be obtained from the motor control system, thus the state of the individual fingers need not be measured. It should be noted that the mechanical design of the finger mechanism ensures that there is no direct relationship between the applied motor torque and the actual finger tip pressure.

The control strategy used at present is achieved by operating the required finger firstly under positional control. On touching an object the positional error will increase, leading to increased applied motor torque. On detection of this condition, the controller will become a torque limited system and by control of the applied torque satisfactory grip control can easily be achieved. If the object moves within the hand as the manipulator is repositioned the fingers will adjust to achieve a stable grip. As the whole arm manipulator is a tele-operated manipulator the visual feedback to the operator is important to the overall control strategy.

\subsection{The finger touch sensor}

In order to determine the absolute force being applied by the finger touch, sensors have been developed and built into the hand at the following points:

(1) Two sensors in the thumb.

(2) One sensor in each finger tip, with the exception of the fourth finger.

(3) Six sensors on the upper surface of the palm. The specification for the touch sensor was determined with reference to the operating requirements of the hand. The design of the hand placed size and environmental constraints on the sensors and hence the enabling technology of the sensors. A range of technologies were investigated including optical, piezoelectric materials, compressible resistive foam and magnetic sensing devices. As a result of this study and the construction of a number of prototypes an optical reflective touch sensor was developed.

The final design is shown in Fig 9. A miniature optical transmitter and receiver package is mounted within a ' $U$ ' shaped spring. The principle of operation is that the amount of reflected light 
depends upon the distance between the optical device and the reflecting surface. The actuation of the sensing element is via a cover plate assembly, the force is transmitted to the spring by a point contact. The output from the sensor is conditioned by an electronic circuit, before being fed to the analogue-to-digital converter. The conditioning system has offset and gain controls to allow calibration of the transducer assembly. Results have that the design has good linearity and low hysteresis. The sensing units have been integrated within the hand and proved to be highly reliable.

\subsection{Implementation of the control strategy}

The three hand motors are controlled by individual proprietary microprocessor drive cards. These cards are capable of controlling the motors in terms of speed, position or torque. The demands of the motors and the mode of operation are determined by the system controller as the result of the commands selected by the operator and the present status of the hand, determined from the drives and the sensors.

The operator has control of the hand from a remote pendant. The controls used by the oper- ator consist of a number of push buttons, six for the three configuration and their respective relaxation modes and two to allow the operator to increase or decrease the applied grip force, based on a knowledge of the task.

The operation of the hand can be considered by reference to the state diagram (Fig. 10). The initial conditions are set during the datum routine normally carried out after power on. Once the hand is at its home position one of the three grip options can be selected (touch, pinch or grip). The control system will then command the required digit(s) to move at a predefined speed and/or torque until an object has been touched. Once a mode has been selected, it is not possible to select any other mode unless the initial selection has been returned to the relaxation state.

The initial selection of the relax option will cause the respective drive(s) to enter the "servo hold' mode in which the digit(s) will hold position irrespective of any external force. A second selection of the respective relax option will cause the 'motor off' mode to be entered, in which power is removed from the corresponding motors. The third and final selection will cause the corresponding motors to move to the initial home position. Once

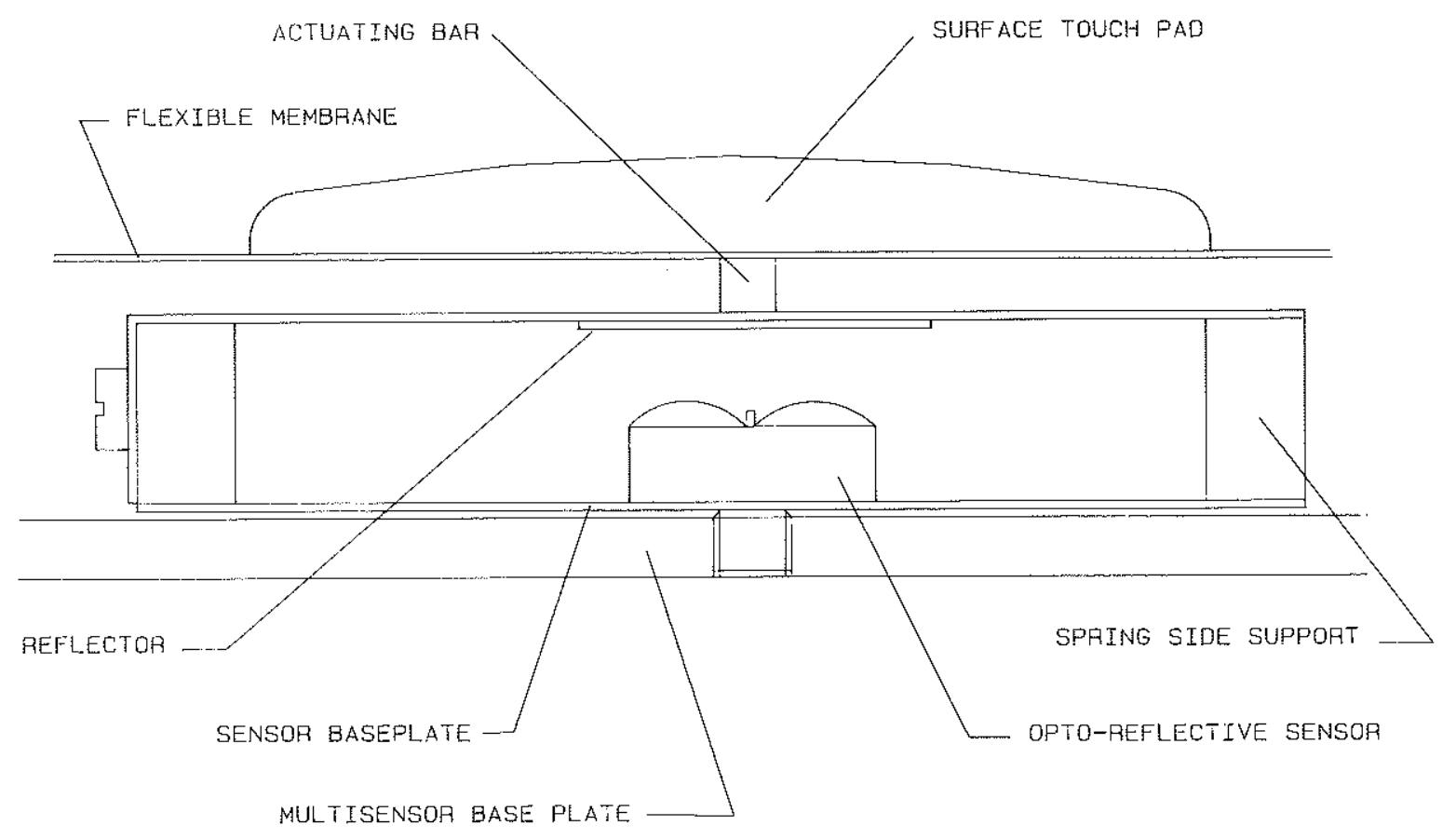

Fig. 9. Outline of the touch sensor mounted in the palm of the hand. The finished unit is approximately $2 \mathrm{~cm}$ long. 


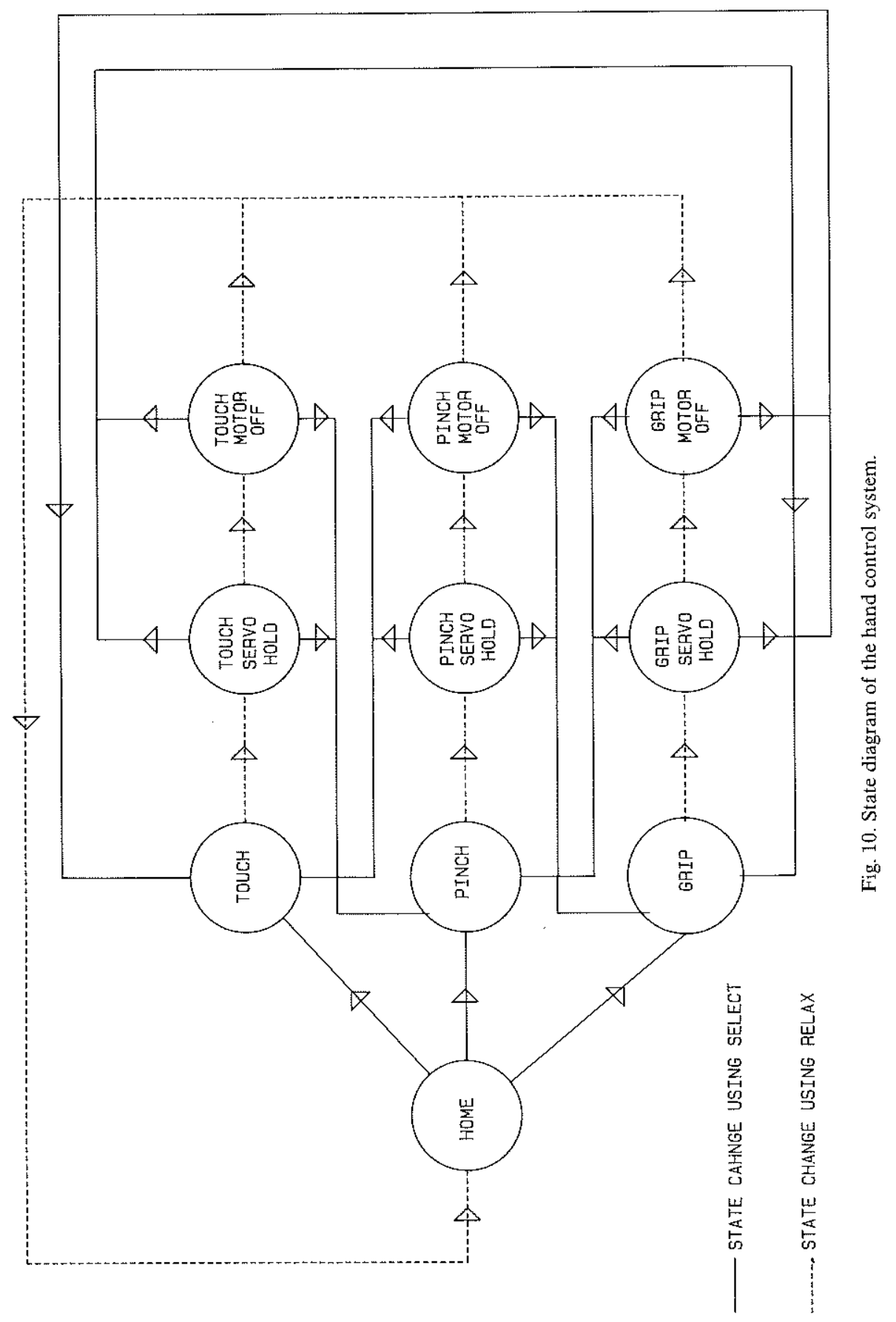


a relaxation mode has been set it is possible to select any of the three hand closure options. In moving between modes the digit(s) not being used will remain in the relaxation state previously attained.

\section{Performance trials and observations}

Trials have been conducted on the hand to investigate its operational characteristics. As the whole arm manipulator is a tele-operated system and hence certain of the performance indicators are a function of the operator's dexterity and performance.

\subsection{Basic handling tests}

Using a number of standard test objects such as pyramids, cylinders, discs and cubes of various masses, a series of tests were conducted, the result of which are summarised in Table 1 . The criterion
Table 1

Summary of handling trials

\begin{tabular}{lllll}
\hline $\begin{array}{l}\text { Object and } \\
\text { nominal mass }\end{array}$ & Test 1 & \multicolumn{2}{l}{ Test 2 } & Test 3 \\
\cline { 2 - 4 } & & $(1)$ & $(2)$ & \\
\hline Disc $0.1 \mathrm{~kg}$ & $\mathrm{~A}$ & $\mathrm{X}$ & $\mathrm{A}$ & $\mathrm{C}$ \\
Disc $0.2 \mathrm{~kg}$ & $\mathrm{~A}$ & $\mathrm{X}$ & $\mathrm{A}$ & $\mathrm{C}$ \\
Disc $0.5 \mathrm{~kg}$ & $\mathrm{~A}$ & $\mathrm{X}$ & $\mathrm{B}$ & $\mathrm{B}$ \\
Cube $0.5 \mathrm{~kg}$ & $\mathrm{~A}$ & $\mathrm{~A}$ & & $\mathrm{~A}$ \\
Cube $1.3 \mathrm{~kg}$ & $\mathrm{C}$ & $\mathrm{C}$ & & $\mathrm{B}$ \\
Cube $3.0 \mathrm{~kg}$ & $\mathrm{X}$ & $\mathrm{X}$ & & $\mathrm{C}$ \\
Rod $0.5 \mathrm{~kg}$ & $\mathrm{~A}$ & $\mathrm{X}$ & $\mathrm{A}$ & $\mathrm{A}$ \\
Rod $1.3 \mathrm{~kg}$ & $\mathrm{~A}$ & $\mathrm{X}$ & $\mathrm{B}$ & $\mathrm{A}$ \\
Rod $3.0 \mathrm{~kg}$ & $\mathrm{~B}$ & $\mathrm{X}$ & $\mathrm{C}$ & $\mathrm{B}$ \\
Pyramid $0.5 \mathrm{~kg}$ & $\mathrm{~B}$ & $\mathrm{X}$ & & $\mathrm{C} / \mathrm{B}$ \\
Pyramid $1.0 \mathrm{~kg}$ & $\mathrm{~B}$ & $\mathrm{X}$ & & $\mathrm{C}$ \\
Pyramid $3.0 \mathrm{~kg}$ & $\mathrm{X}$ & $\mathrm{X}$ & & $\mathrm{C}$ \\
\hline
\end{tabular}

used to judge the operation is quite broad, but allows for easy assessment of the individual tasks. In addition, the performance of the human operator was used as a bench mark for the trials. Each

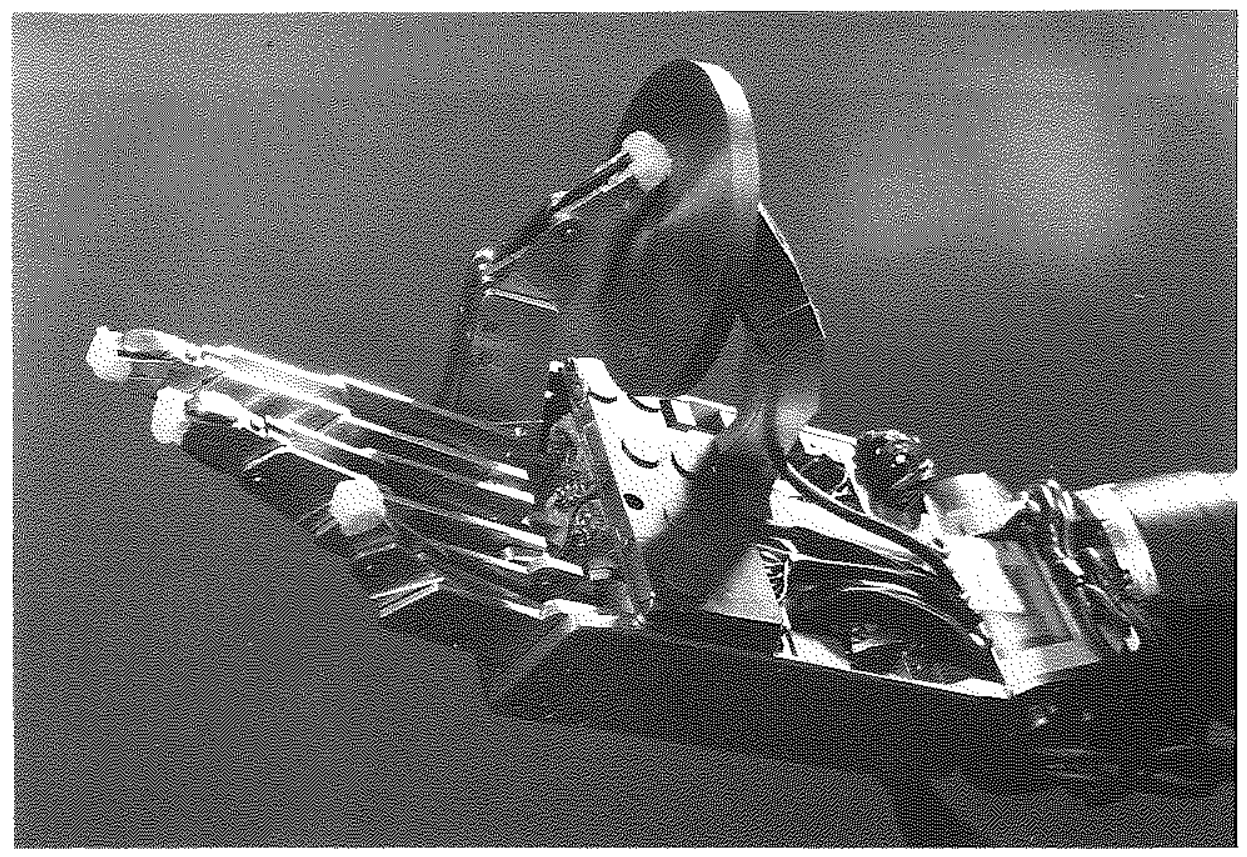

Fig. 11. Optimal pinch position. The silicon rubber at the end of the finger is to prevent damage to the glove, in later tests this was replaced with rubber finger tip covers. 
task is graded on a scale $\mathrm{A}$ to $\mathrm{C}$, with $\mathrm{X}$ indicating that the task is impossible. In test 1 the object was placed by an operator directly into the hand. Test 2 measures the ability of the hand to directly grasp an object from a flat surface, column 1 is when the object was flat on the surface, column 2 is when one edge was raised above the surface. Test 3 was with a skilled operator performing the same handling tasks.

\subsubsection{Discs}

Due to the limited thickness of the discs it was not possible to pick them up directly from the table top, when flat, largely due to the rigidity of the finger tips. It is necessary to raise the disc from the table by some means and then pinch the disc. One possible solution is for the base of the glove box to have a corrugated or equivalent surface.

Fig. 11 shows the optimum pinching position, with the smallest disc. It was found that with the hand inserted into the glove the position shown in Fig. 12 gave the most satisfactory results. This posture also results in the least strain on the glove, an important consideration to prevent the glove from being torn.

\subsubsection{Cubes}

The $0.5 \mathrm{~kg}$ and $1.3 \mathrm{~kg}$ cubes could be picked up in all tests. Care has to be taken in the orientation of the whole arm manipulator to ensure that satisfactory grip points on the cubes' faces were being used. However, with the $3.0 \mathrm{~kg}$ cube the span required is in excess of that achievable by the manipulator without causing damage to the glove. It should be noted that this object is difficult for a human operator to manoeuver.

\subsubsection{Cylindrical rods}

It is not difficult for a human operator to pick up this object. The whole arm manipulator again needs a raised edge to get a full power grip around the object. Again this is largely due to a lack of compliance in the manipulator's finger tips. Fig. 13 shows the manipulator holding a rod in the cylindrical power grip.

\subsubsection{Pyramids}

This shape poses problems in that there are no faces capable of providing a gripping surface for a direct lift. A human will pick up the pyramid by pinching and rely on the surface friction and the

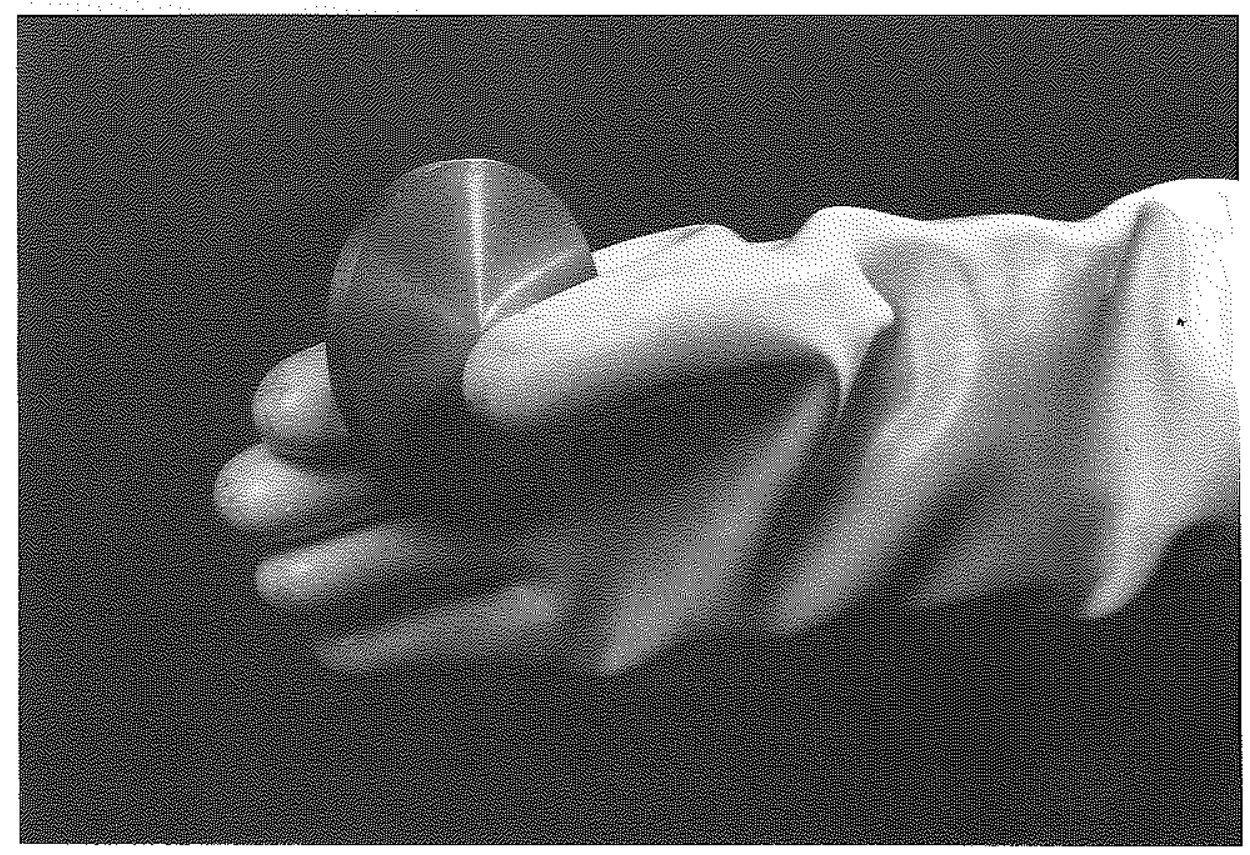

Fig. 12. The whole arm manipulator gripping the $0.5 \mathrm{~kg}$ disc. 


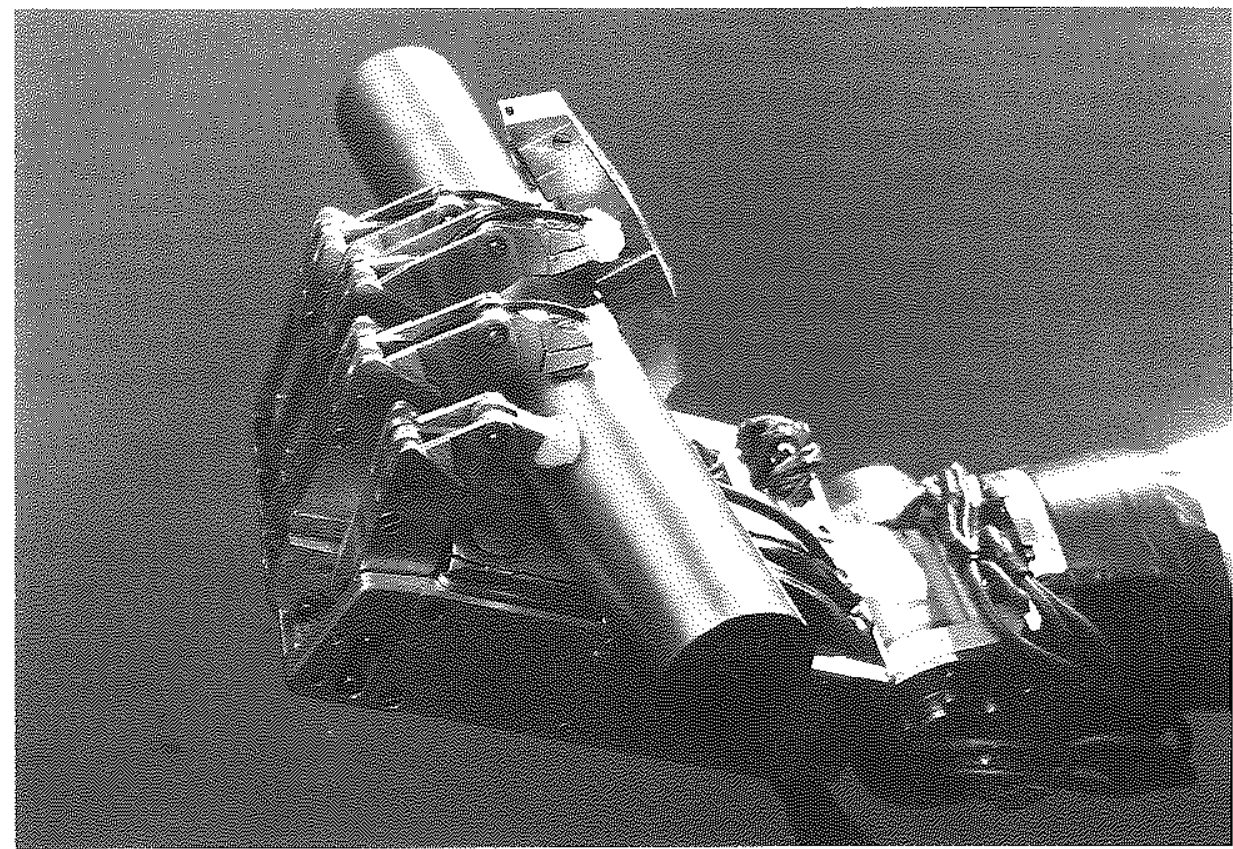

Fig. 13. The $1.5 \mathrm{~kg}$ rod being held in the cylindrical power grip. The sensors can be clearly seen on the thumb, second and third finger.

compliance of the finger tips; with the manipulator this is not possible.

\subsection{Observation relating to the operation of the touch sensors}

The handling trials discussed above were conducted with the hand in either a side-on or palmdown orientation. The resultant grip in these conditions was usually a pinch between the thumb and index finger. It was found that there was little or no contact with the palm plate sensors. As a result, additional tests were conducted to study the location of objects when grasped relative to the sensors.

In a gloveless hand, the centre of the power grip for an object is equidistant from the finger pivots to the thumb pivot, this is clearly seen in Fig. 13. The effect of placing the hand in a glove is to move this point towards the fingers and away from the palm plate area. The presence of the glove stretching prevents the object touching sensors located to the rear of the palm. This movement of the object also affects the lower thumb sensor which shows little responce unless the object is forced against the bottom of the thumb, with the resultant additional stretching of the glove material.

It was noted that which sensor responds is largely dictated by the shape and size of the object together with its position in the hand. The same object picked up in a similar grip does not neccessarily cause an identical sensor response. It was concluded that the control of this type of hand should be based on the torque control method. The individual touch sensors are used to provide local information as and when required.

\section{Conclusions}

The development of dexterous hands is considered to be of considerable importance to further the implementation of robotic systems in hazardous environments. The fitting of a dexterous hand ensures that any resonable task can be 
performed by a manipulator without the need for a range of end effectors. The work discussed in this paper has centered on a specific requirement - that of a robot for glove box applications; this required the development of an anthropomorphic hand. The hand discussed in this paper has all the major attributes of the human hand, namely, four fingers and thumb, together with all the actuators within the size profile of the human hand. To achieve this a number of novel mechanisms have had to be developed. The resultant finger motions are closely analogous to those of the human finger.

To achieve full benefit from this unique mechanical hand, a control package has been developed which allows total control of the hand using a small number of high-level commands. The trials that have been conducted have clearly demonstrated that the hand is capable of performing a range of tasks suitable for a tele-operated robot.

\section{Acknowledgements}

The work discussed in this paper was funded by the United Kingdom Ministry of Defence. The author acknowledges the contributions from Professor J. Nightingale and Mr K. Barlow. In addition, the author acknowledges the considerable contribution from Mr D. Whatley of the Central Design Service for the mechanical concepts embodied in, and the detailed mechanical design of the hand. All drawings in this paper were produced by the Central Design Service.

\section{References}

[1] D.S. Childress, Artifical Hand Mechanisms, Proc. Mechanisms Conference and International Symposium on Gearing and Transmission, San Fransisco (1982).

[2] P.H. Chappell, J.M. Nightingale, P.J. Kyberd and M. Barkhordor, Control of a single degree of freedom artificial hand, J. Biomed. Eng. 9 (1987) 273-277.

[3] A. Bejczy, Sensors, Controls and man-machine interface for advanced teleoperation, Science 208 (1980) 1327-1335.

[4] S.C. Jacobsen, J.E. Wood, D.F. Knutti and K.B. Biggers, The Utah/MIT hand-Work in Progress, Robotics Research (I It International Symposium) (MIT Press, Cambridge, MA, 1984).

[5] M.T. Mason and J.K. Salisbury, Robot Hands and the Mechanics of Manipulation (M1T Press, Cambridge, MA, 1985).

[6] R.M. Crowder, An Whole Arm Manipulator for Hazardous Environments, $10^{t h}$ B.R.A. Conference, Birmingham (1987).

[7] G. Schlesingert, Der Mechanische Aufbau der künstlichen Glieder, Ersatzglieder und Arbeitshilfen, Part 2 (Springer, Berlin, 1919).

[8] F.R.E. Crossby and F.G. Umholtz, Design of a three fingered hand mechanism, Mech. Mach. Theory 12 (1977) $85-95$.

[9] R.D. Codd, Development and evaluation of adaptive control for a hand prosthesis, University of Southampton Ph.D. Thesis, 1975.

[10] D. Moore, Development of a Multifunction Adaptive Hand Prosthesis, University of Southampton Ph.D. Thesis, 1980.

[11] Sensors-Touch, Force and Torque, International Encyclopedia of Robotics (John Wiley, New York, 1988). 